\title{
Distress, anxiety, depression, and emotional well-being in African-American men with prostate cancer
}

\author{
Christian J. Nelson*, Eliana M. Balk and Andrew J. Roth \\ Department of Psychiatry and Behavioral Sciences, Memorial Sloan-Kettering Cancer Center, New York, NY, USA
}

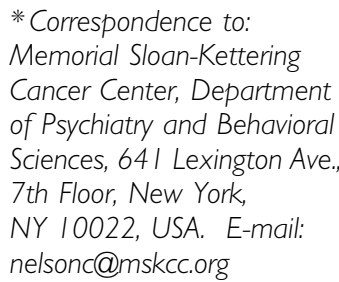

Received: 30 March 2009

Revised: 24 September 2009

Accepted: 29 September 2009

\begin{abstract}
Objective: African-American men have an incidence rate of prostate cancer $60 \%$ higher than Caucasian men. Over one-quarter of men with prostate cancer experience significant distress, yet psychosocial research has rarely focused on African-American men. This study presents novel data on emotional well-being, distress, anxiety, and depression in African-American men with prostate cancer.

Methods: This archival research combined two databases $(N=385$ and $N=367)$ comprised of 55 African-American men with prostate cancer. Quality of life was assessed with the Functional Assessment of Cancer Therapy, distress was measured with the Distress Thermometer, and anxiety and depression were measured with the Hospital Anxiety and Depression Scale. African-American and Caucasian men were matched on age, education, and stage of disease, and compared on emotional well-being, distress, anxiety, and depression.

Results: The mean age of the 55 African-American was 63 years old. In non-matched comparison, African-American men had elevated levels of distress, anxiety, and depression similar to Caucasian men. African-American men reported high levels of clinically significant distress $(>31 \%)$ and anxiety $(>23 \%)$. However, after matching the African-American and Caucasian men, African-American men reported higher mean scores on emotional well-being $(p<0.05)$ and a lower percentage of African-American men displayed clinically significant depressive symptoms $(p<0.05)$ compared with Caucasian men.

Conclusions: After matching the sample, African-American men seem to display a sense of resilience, demonstrating greater emotional well-being and a lower incidence of clinically significant depressive symptoms, compared with Caucasian men. This is consistent with crosscultural research outside of prostate cancer. Continued research is needed to further elucidate the concept of resiliency in African-American men with prostate cancer.

Copyright (C) 2010 John Wiley \& Sons, Ltd.
\end{abstract}

Keywords: cancer; oncology; African-American; Distress; prostate

\section{Introduction}

Prostate cancer is the most common site of cancer in men in the United States, with over 180,000 men projected to be diagnosed in 2009 [1], and ranks as the second leading cause of death in men in the United States [1]. Not surprisingly, a significant number of men with prostate cancer experience anxiety and distress at some point when dealing with this illness. In a study of 121 mostly advanced stage prostate cancer patients, $32.6 \%$ of men scored at or above the anxiety cut-off score of the Hospital Anxiety and Depression Scale (HADS) [2], and $15.2 \%$ scored at or above the cut-off score for depression on the HADS [3]. Zabora et al. (2001) found, similarly, in a study of psychological distress by cancer site, that $30.5 \%$ of 167 prostate cancer patients had significant psychological distress based on a self-report rating scale, the Brief Symptom
Inventory (BSI) [4,5], consistent with the estimates that $25-30 \%$ of all cancer patients meet diagnosis criteria for a major depressive disorder or an anxiety disorder. [6-9]. Some causes of the anxiety found amongst these men with prostate cancer include worries about side effects of their medical treatment, metastasis of their disease, disability, death, and 'PSA Anxiety' [10], the anxiety associated with Prostate Specific Antigen (PSA) testing.

African-American men are particularly vulnerable towards prostate cancer with an incidence rate that is $60 \%$ higher than Caucasian men [11]. African-American men have a greater likelihood of being diagnosed with advanced cancer [12] and 2.3 times greater likelihood of dying from the disease as compared with Caucasian men [11]. African-American men are also more likely to be diagnosed at a younger age [13]. These factors are compounded by the reality that African-American 
men are less likely to have appropriate knowledge of the risks of prostate cancer [14-18] or to have a digital rectal exam $[19,20]$.

These realities for African-American men may impact how they cope with their disease. To illustrate, Abrams et al. (1990) suggest that age may be the most important factor that determines a person's psychological response to a diagnosis of cancer, and younger men with school age children may react very differently than a relatively older man with adult age children [21]. Similarly, the stage of diagnosis can also have a distinct impact on how a person reacts to a cancer diagnosis or treatment [22], and if African-American men are diagnosed with later stage disease they may be experiencing more distress as a result. Despite these statistics however, psychosocial research undertaken in men with prostate cancer generally lacks appropriate sampling of African-American men, and research that focuses specifically on distress, anxiety, and depression in African-American men with prostate cancer is quite limited [21].

The primary psychosocial research conducted in African-American men with prostate cancer has focused on general quality of life measures. This initial research suggests that African-American men score lower on quality of life measures as compared with Caucasians. In a study comprised of 958 Caucasian and 161 African-American men newly diagnosed with prostate cancer, which controlled for age, education, and income level, Lubeck et al. (2001) found that African-American men had lower quality of life ratings on almost all health specific and general dimensions on the SF36 at baseline and follow-up assessments [23]. In addition, while quality of life of both AfricanAmerican and Caucasian men improved a year after follow-up, African-American men's worry about their disease improved at a slower rate than Caucasians. Also, Caucasian men reported better self-esteem, emotional, and mental health than African-American patients at both the 3 and 12-month follow-up. Jenkins et al. (2004) examined the quality of life and impact of cancer on sexuality in a sample of men from the Cleveland Clinic database [24]. From a sample of 1, 112 Caucasian men and 118 African-American men who averaged 4.5 years past localized treatment [24], AfricanAmerican men reported slightly lower scores on mental health (two points), were less likely to report an impact on erections or sexual satisfaction, and were more likely to report a loss of libido.

Together these studies imply that quality of life is lower in African-American men with prostate cancer; however, these studies do not address incidences of distress, depression, or anxiety specifically. This is not necessarily surprising as there is often general lack of clarity in the literature about what is meant by quality of life constructs such as 'emotional well-being' or general psychological constructs such as 'distress'. Distress is generally considered to be a combination of feelings such as worry, anxiety, fear, and depression. The most common assessment of distress in cancer patients is an efficient one-question screen (i.e. The Distress Thermometer) that is used to determine if further evaluation should be considered [25]. Emotionalwell being is commonly assessed within quality of life measures, and like distress, also combines the constructs of anxiety and depression [26]. Emotional well-being scales are usually longer (around 4 or 5 questions) and are considered more psychometrically sound as compared with the one-question distress measures. Although these concepts and scales are useful for identifying general distress, they lack the ability to make the distinction between anxiety and depression. We believe this distinction is important in order to assist clinicians to better understand and treat psychological difficulties in this population.

The current study, therefore, aims to elucidate the incidence of these markers of psychological distress, anxiety, and depression as well as emotional wellbeing within African-American men with prostate cancer, and in so doing to contribute to the dearth of scientific literature in this area. To do this, we used archival data including measures of depression, anxiety, and emotional well-being from 752 men with prostate cancer generally divided equally between early and late-stage disease. We hypothesized based on the studies listed above where African-American men with prostate cancer reported poorer quality of life, that African-American men would report higher levels of distress, anxiety, and depression, and lower levels of emotional wellbeing compared with Caucasian men.

\section{Methods}

\section{Participants}

The study utilized two separate samples, both investigating quality of life, distress, anxiety, and depression in men with early and late-stage prostate cancer. The first database consisted of data from 385 men from doctors' offices throughout the US. The average age for the participants was 71 year old ( \pm 9 years). The majority were Caucasian $(86 \%)$, and $10 \%$ were African-American. Roughly half had early stage disease $(55 \%)$ and the remainder has late-stage disease. The average time since diagnosis was 4.4 years $( \pm 4)$. A large percentage $(42 \%)$ had a college or advanced degree. The majority were married or in a committed relationship (83\%). The second database was based on 367 men from the prostate clinics at our institution. The average age from this sample was 66 years old ( \pm 9 years). The majority of participants were Caucasian $(89 \%)$, while $6 \%$ were African-American. Almost half 
$(45 \%)$ had early stage disease, and the remainder had late-stage disease. The average time since diagnosis was 5.2 year $( \pm 4.5)$. A majority $(73 \%)$ had earned a college degree or higher. The majority $(84 \%)$ were married or in a committed relationship.

When combining both databases a total of 752 subjects comprised the total sample. When accounting for 29 subjects who had missing data on all the variables utilized in this analysis, a total number of 723 were available for analysis. We were specifically interested in two subgroups for analysis in this paper; the $55(8 \%)$ African-American men available in the data pool, and the $631(87 \%)$ Caucasian men as a comparison group. When combining the Caucasian and African-American men, there were 686 subjects used for this analysis. The remaining $35(5 \%)$ subjects were identified as Hispanic (non-African-American), Asian, or 'other' ethnic background. We did not include Hispanics (nonAfrican-American), Asian, or 'other' ethnic identities in the comparison group with the Caucasian men because these groups are unique cultures that may have their own ethnically specific ways of dealing and coping with stressful situations.

To control for specific variables, we matched an equal sample of Caucasian men to the AfricanAmerican men. We selected to match the samples on age, education (college graduate vs not college graduate), and stage of disease (early vs late). One African-American subject did not have this demographic data recorded, and as a result we matched 54 Caucasian men to 54 African-American men. As experimental control is fundamentally distinct from statistical control, we elected to match subjects on these variables as opposed to controlling for them statistically [27]. We recognize that it is always important to understand that the larger sample may vary systematically on the variables that we used to match, however, this helps to understand the differences between the matched Caucasian sample and the non-matched Caucasian sample. Age and stage of disease were chosen because data suggests that African-American men are diagnosed at a younger age with later stage disease, and these variables may have an impact on the amount of distress men experience as a result of prostate cancer (please see introduction). Socioeconomic status (SES) is also an important variable where there may be differences between AfricanAmerican men and Caucasian men, and these differences may impact distress [28]. However, as we did not have socio-economic data, education was selected as a proxy for SES. Although not ideal, education is considered a relatively good proxy for SES [27].

\section{Procedure}

The eligibility requirements for both studies included English fluency, diagnosis of prostate cancer, age greater than 18 years, and monitoring of prostate serum antigen (PSA) levels. Patients were recruited consecutively as they came to their appointments with their prostate cancer physician. One goal of recruitment was to recruit an equal number of men with early and late-stage disease. Patients who met the eligibility criteria were asked to complete a series of seven self-report questionnaires as they waited for their doctor's visit. The questionnaires were completed before they received the results of their PSA test for that specific visit. Data from the following questionnaires were analyzed in this study: The Distress Thermometer [3], the HADS [29], the Functional Assessment of Cancer Therapy (FACT-G) [30,31], and a demographics questionnaire that included treatment course and co-morbidities.

\section{Measures}

The Distress Thermometer is a 1-item visual analog scale (picture of thermometer), which asks patients to rate their distress on a $0-10$ scale. This scale has demonstrated good convergent and discriminant validity in studies with cancer patients as compared with longer measures used to screen for distress $[32,33]$ Research suggests that a cut-off of 4 or greater warrants referral for mental health assessments [32].

Depression and anxiety were measured by the HADS [2]. This is a 14-item self-report questionnaire, which has been well tested in cancer populations. Responses are scored on a 4-point scale, and the measure is considered particularly useful for patients with chronic diseases because of the absence of somatic items that often confound the determination of psychiatric problems among the medically ill. As its name implies, the HADS has a depression and anxiety subscale, each consisting of 7 items. The HADS has demonstrated strong test-retest reliability in both elderly patients and patients with AIDS [34,35]. A number of cutoff scores for the HADS have been defined which indicate significant depression or the need for referral in cancer patients [36]. As none of these studies have specifically validated the cut-off scores in men with prostate cancer or African-American men with prostate cancer, we elected to use a more stringent cut-off for the total HADS score of $\geqslant 16$, and $\geqslant 8$ for the two subscale scores, consistent with research that found a cut-off of 8 to be optimal for lung cancer patients [2,37-39]. The analysis used HADS scores as both a continuous variable and a dichotomous variable, as we felt the continuous variable would tap levels of depressive symptoms and we were interested in a cut-off score that would be more appropriate at defining significant depression. As such, we opted to use the higher cut-off scores for this study.

Emotional well-being was measured with the emotional well-being subscale of the FACT-G 
[30,31]. The FACT-G is a quality of life questionnaire which includes a 27 -item 'core' quality of life measure grouped into four subscales: physical well-being, social/family well-being, emotional well-being, and functional well-being. The FACT$\mathrm{G}$ items are rated on a 5-item Likert scale, from 0 , 'not at all' to 4, 'very much'. The internal consistency of the subscales of the FACT-G range from acceptable to excellent, from 0.65 to 0.82 . The total FACT-G demonstrates excellent internal consistency with an alpha coefficient of 0.89 . The test-retest reliability of the FACT-G is also excellent within a 7-day period, with correlations ranging from 0.82 to 0.92 [30].

\section{Statistical analysis}

Descriptive statistics were reported for both the African-American sample and the Caucasian sample. Results for both matched and non-matched Caucasian samples were included for analysis in order to show that without proper variable control, results can potentially be misleading. Independent measures $t$-tests were used when examining the differences on continuous variables between the groups in the non-matched sample. Matched samples $t$-tests were used when examining the difference in continuous variables between the groups in the matched sample. The percentage of each group reporting significant distress, depression and anxiety were reported (please see above for the specific cut-off scores used). The difference in percentages between groups was tested using Chi-Square. As this is archival data, we calculated the effect sizes for mean comparisons needed to have an $80 \%$ chance of finding significance with a two-tailed test alpha level 0.05 . For the nonmatched sample, differences would generally need to reach a Cohen's $d$ of 0.40 , and for the matched sample differences would need to reach a Cohen's $d$ of 0.55 .

\section{Results}

\section{Participant characteristics}

From the combined databases consisting of 686 subjects, there were 631 Caucasian men with a mean age of $69( \pm 10)$ years. About half $(51 \%)$ had late-stage disease. The average time since diagnosis was 4.9 years $( \pm 4.4)$. Eighty-four percent were married or in a relationship and $60 \%$ had a college education or higher. The mean age of the 55 African-American men was $63( \pm 10)$ years old and $53 \%$ had late-stage disease. The average time since diagnosis was 4.8 years $( \pm 4.3)$. Seventy-nine percent were married or in a relationship and $41 \%$ had a college degree or higher.

When comparing African-American men and Caucasian men on demographic variables, there was no significant difference in disease stage, time since diagnosis, or marital status. However, African-American men were younger (63 vs 69, $p<0.01$ ), and a lower percentage had completed a college education or higher ( 41 vs $60 \%, p<0.01)$ as compared with the Caucasian men.

Levels of distress, anxiety, depression, and emotional well-being in non-matched subjects

When comparing the African-American men to the Caucasian men, African-American men generally reported levels of distress, anxiety, depression, and emotional well-being similar to Caucasian men with prostate cancer. On the Distress Thermometer, there were no significant mean differences between African-American men and Caucasians (See Table 1). When assessing the number of men meeting the cut-off score of $\geqslant 4$, although a greater percentage of African-American met this cut-off compared with Caucasian men (32 vs $23 \%$, $p=0.12, \mathrm{RR}=1.39$, see Table 2), this difference did not meet statistical significance levels.

When analyzing the HADS scores, AfricanAmerican men tended to report similar results compared with Caucasian men, with the exception of one result with those men meeting the cut-off on the HADS anxiety subscale. There were no mean differences between African-American men and Caucasian men in total HADS, HADS depression scores, or HADS anxiety scores (see Table 1). When analyzing the number of men scoring above the HADS cut-off scores, there was no significant difference in the number of African-American and Caucasian scoring $\geqslant 16$ of the HADS total scores, or scoring $\geqslant 8$ on the HADS depression subscale. However, on the HADS anxiety subscale, AfricanAmericans reported a trend towards a significantly higher percent at the $\geqslant 8$ cut-off, $24 \%$ as compared with $15 \%$ of Caucasian men $(p=0.09, \mathrm{RR}=1.6$, see Table 2).

When comparing the mean levels on the Emotional Well-Being subscale of the FACT, there was no significant difference between African-American men and Caucasian men (See Table 1).

Table I. Mean differences comparing the African-American sample to the non-matched Caucasian sample

\begin{tabular}{lrrrrrrr}
\hline Variables & $\begin{array}{c}\text { African- } \\
\text { Americans }\end{array}$ & & \multicolumn{2}{c}{$\begin{array}{c}\text { Caucasians non- } \\
\text { matched sample }\end{array}$} & p & d \\
\cline { 2 - 3 } & Mean & SD & & Mean & SD & & \\
\hline Distress Thermometer & 2.19 & 2.26 & & 2.13 & 2.25 & 0.86 & 0.03 \\
HADS Total & 7.05 & 5.96 & 6.82 & 5.61 & 0.77 & 0.04 \\
HADS Anxiety & 4.26 & 3.77 & & 3.90 & 3.37 & 0.45 & 0.11 \\
HADS Depression & 2.79 & 2.75 & 2.92 & 2.97 & 0.75 & 0.04 \\
Emotional well-being & 20.16 & 3.19 & & 19.65 & 3.87 & 0.33 & 0.14 \\
\hline
\end{tabular}


Levels of distress, anxiety, depression and emotional well-being in a matched sample

After matching the African-American and Caucasian men on age, education, and stage of disease (see above), African-American men continued to display similar levels of distress as compared with Caucasian men on the Distress Thermometer. There was no significant mean difference between African-American men and Caucasians nor were there significant differences between African-American men vs Caucasian men when assessing the percent of men meeting the cut-off score of $\geqslant 4$.

When analyzing the mean HADS total and subscale scores, differences did not meet statistically significance levels; however, African-American men consistently demonstrated lower scores with small effects sizes (i.e. Cohen's $d$ approximately 0.2 , see Table 3 ). The small effect sizes indicate that a larger sample would be needed to demonstrate statistical significance, and this archival data set was not powered correctly to detect these differences.

When assessing the percentage of men scoring at the cut-off of $\geqslant 16$ for HADS total scores, although the actual percentage difference is large with lower percentage of African-American men scores below this cut-off compared with Caucasian men, there was no significant difference ( 9 vs. $19 \%, p=0.17$, $\mathrm{RR}=2.11)$. There was also no significant difference between African-American men and Caucasian men on the percent of men scoring $\geqslant 8$ on the

Table 2. Comparisons of percent of patients who met cut-off scores

\begin{tabular}{lcccc}
\hline Variables & Cut-off & $\begin{array}{c}\text { African- } \\
\text { American }\end{array}$ & $\begin{array}{c}\text { Caucasian non- } \\
\text { matched sample }\end{array}$ & $p$ \\
\cline { 3 - 5 } & & $\%$ & $\%$ & \\
\hline Distress Thermometer & $\geqslant 4$ & 33 & 23 & 0.12 \\
HADS total & $\geqslant 16$ & 9 & 8 & 0.82 \\
HADS Anxiety & $\geqslant 8$ & 24 & 15 & 0.09 \\
HADS Depression & $\geqslant 8$ & 4 & 8 & 0.22
\end{tabular}

Table 3. Mean differences comparing the African-American sample to the non-matched and matched Caucasian samples

\begin{tabular}{|c|c|c|c|c|c|c|}
\hline \multirow[t]{2}{*}{ Variables } & \multicolumn{2}{|c|}{$\begin{array}{c}\text { African- } \\
\text { Americans }\end{array}$} & \multicolumn{2}{|c|}{$\begin{array}{c}\text { Caucasian } \\
\text { matched } \\
\text { sample }\end{array}$} & \multirow[t]{2}{*}{$p$} & \multirow[t]{2}{*}{ d } \\
\hline & Mean & SD & Mean & SD & & \\
\hline Distress Thermometer & 2.15 & 2.22 & 2.60 & 2.34 & 0.33 & 0.14 \\
\hline HADS Total & 7.12 & 5.94 & 8.89 & 6.25 & 0.12 & 0.21 \\
\hline HADS Anxiety & 4.24 & 3.78 & 5.28 & 3.88 & 0.16 & 0.19 \\
\hline HADS Depression & 2.84 & 2.74 & 3.61 & 3.25 & 0.16 & 0.18 \\
\hline Emotional well-being & 20.28 & 3.04 & 18.14 & 4.56 & $<0.01$ & 0.44 \\
\hline
\end{tabular}

The means for African-American subjects changes slightly from Tables I and 3 because I African-American subject did not report demographic data and could not be matched.
HADS anxiety subscale (24 vs. $26 \%, p=0.82$, $\mathrm{RR}=1.08$ ); however, a significantly lower percentage of African-American men met the cut-off of $\geqslant 8$ on the HADS depression subscale compared with Caucasian men ( 4 vs. $15 \%, p=0.04, \mathrm{RR}=3.75)$.

When comparing the mean scores of the Emotional Well-Being scale on the FACT, AfricanAmerican men reported significantly higher emotional well-being compared with Caucasian men $(20.28 \pm 3.04$ vs $18.14 \pm 4.56, p<0.01, d=0.44$, see Table 4).

\section{Discussion}

This study aimed to answer the question of whether there are differences in distress, anxiety, depression, and emotional well-being between cohorts of African-American and Caucasian men with prostate cancer. Based on results from limited research in this area, we hypothesized that African-American men would consistently report higher levels of distress. Interestingly, however, we found that African-American and Caucasian men reported distress at similar levels in an unmatched sample, with the exception that African-American men showed a trend towards higher anxiety. When comparing the African-Americans men to a Caucasian sample matched on age, education, and stage of disease, a lower percentage of AfricanAmerican men reported clinically significant depressive symptoms (i.e. $\geqslant 8$ on the HADS depression subscale) and greater mean scores on emotional well-being. There were no significant differences in mean scores on HADS total scores, or the depression and anxiety subscales on the matched-samples; however, African-American men consistently reported lower mean scores with a Cohen's effect size of approximately 0.2 , indicating a small effect. Unfortunately, this archival study had power to only detect medium effects, and did not have sufficient power to detect these small effects as statistically significant. One value of archival data is to help guide future research, and indeed, the National Cancer Institute values preliminary findings from secondary analysis to justify potential research. Taking this into account, we do

Table 4. Comparisons of percent of patients who met cut-off scores

\begin{tabular}{lccccc}
\hline Variables & Cut-off & $\begin{array}{c}\text { African- } \\
\text { American }\end{array}$ & $\begin{array}{c}\text { Caucasian } \\
\text { matched sample }\end{array}$ & $p$ \\
\cline { 3 - 3 } & & $\%$ & & $\%$ & \\
\hline Distress Thermometer & $\geqslant 4$ & 33 & & 25 & 0.39 \\
HADS Total & $\geqslant 16$ & 9 & & 19 & 0.17 \\
HADS Anxiety & $\geqslant 8$ & 24 & & 26 & 0.82 \\
HADS Depression & $\geqslant 8$ & 4 & & 15 & 0.04 \\
\hline
\end{tabular}


not view these as 'null' findings, but instead view the consistency of these differences (and consistency with the literature discussed below) as markers for future research, and have provided the effects sizes to help guide appropriate sample size calculations for subsequent studies.

As there is a general dearth of literature of distress in African-American men with prostate cancer, it is difficult to place the results of this study in context with other studies of distress in this population. As a result, we will review relevant literature on African-Americans conducted outside of prostate cancer. Our results, while surprising, appear to be consistent with previous research in healthy samples which indicates that before controlling for important confounding variables when comparing African-Americans to other ethnicities, African-Americans appear to report greater distress. However, after controlling for important variables, as we did matching the samples on age, education, and stage of disease, African-Americans report reduced distress [28, 40-42]. For example, in Sachs-Ericsson's study, which compared results of Caucasian and African-American elders' responses on the CES-D, African-Americans first appeared to endorse more depressive symptoms than Caucasians, but this effect was mediated when socioeconomic factors were controlled and whites actually endorsed more depressive symptoms. The authors cite studies that show that Caucasians have lower self-esteem than AA, a racial difference that increases with age $[43,44]$. They also cite studies of caregivers that found that Africans-Americans experience less distress and depressive symptoms $[45,46]$ and that African-Americans have better interpersonal functioning, which protects them against depressive symptoms [47].

Studies that have found differences between African-Americans' and Caucasians' emotion regulation and affective style may also partly explain why African-Americans report less depression. The medical anthropological literature shows that certain cognitive and emotional descriptors of depression are absent in some groups [48]. Consedine et al. found that African-American and Caribbean women scored lower than other ethnic groups on a self-report measure of anger in a covariate analysis of a multi-ethnic group of women $(N=1364)[49,50]$. Several other studies have also shown that African-Americans have lower emotional expression and lower levels of negative affect [51] that may explain why AfricanAmericans would be less likely to endorse depressive symptoms. In addition, Steward et al. [52] found that African-Americans have a greater reliance on humor, which has been shown to be related positively to a number of factors associated with psychological health, such as optimism and self-esteem, and negatively with signs of psychological distress [53].
African-Americans have also been shown to have more extended families and greater social support [54], which together may aid in preventing depressive symptoms. Specifically, African-Americans may have stronger ties to a religious community and participate more actively in religious activities [54] and several studies provide further evidence of the prominence of organized religion and spirituality within the African-American community. AfricanAmericans report relationships with God that are very close and intimate, and are more likely to use spirituality as a coping mechanism for a difficult diagnosis [55]. Indeed, Musick et al. (1998) examined the effect of religious activity on depressive symptomology among Black and White community-dwelling elders with cancer and found that the effects of religious activity were stronger for Blacks than Whites, and that among Blacks, religious activity was related to lower levels of depressive symptomology [56].

Another way to frame this discussion is to focus on the concept of 'resilience'. Resilience is defined by some authors as the reporting of lower psychological symptoms [57], while other authors have attempted to define resilience as a distinct concept and have developed specific assessments to measure this as a distinct outcome variable. The research exploring resilience in African-Americans tends to be limited and focuses on the reduction of psychological symptoms. As such, this literature reported similar finding as discussed above, and has found that cultural, socio-familial, and psychological resources served to inhibit psychological distress in African-Americans exposed to stressful life events [57]. As one study suggests, this resilience may actually be present in African-Americans not in spite of racism, but because of it. In a 10-year qualitative investigation of older African-Americans' experiences with chronic illness, Becker and Newsom (2005) found that African-American consistently reported themes of independence, spirituality and survival, themes of resilience that the authors attribute to African-American's ongoing confrontation with racism [58].

While these explanations of lower levels of depression within the African-American community are encouraging, it is important to consider them within the context of mediating factors that may distort actual levels of depression within the African-American community. Research has shown that African-Americans are less likely to seek mental health care than Caucasians [59]. A study by Anglin et al. (2008) offers a possible explanation to this discrepancy; they found that African-Americans are more likely than Caucasians to believe that mental health problems improve on their own, without professional intervention [60]. Even more tellingly, Cruz et al. (2008) found that shame and denial were cited by African-Americans as their primary barriers to seeking care for depression [61]. Therefore, while 
increased social support and religious participation may indeed inhibit depression, perhaps the stigma towards depression in the African-American community distorts the accuracy of self-report measures of depression within any African-American sample. Likewise, the studies cited earlier that have found differences in African-Americans' emotional and affective style acknowledge that it is unclear whether these differences are due to resiliency towards life challenges or to denial of them. Finally, it is important to note that while African-Americans scored lower on the depression subscale of the HADS, the fact that they scored similar to Caucasians on the anxiety subscale indicates that African-Americans are certainly not immune to distress; they simply may display it differently.

We believe that there are several important strengths of this study. Given the lack of data on distress, anxiety, depression, emotional well-being, or resilience in African-American men with prostate cancer, coupled with the distinct significance to focus on this population, we believe that is novel data and important to help us to begin to understand the experiences of African-American men with prostate cancer as well as generate hypotheses for future research. We also view the broad range of prostate cancer patients (e.g. mix of late and early stage, and varied treatment history) as a strength providing an overview of men's experience and laying the foundation that will drive future research. However, we recognize that this nonspecificity may be viewed as a weakness of the study, and acknowledge the need for future studies that are more narrow in scope on the stage of disease or treatment history. We also concede that there are several other limitations to this study. Because the study was conducted through archival data, we were limited to a relatively small sample of African-American men and we do not have data on important variables such as measures of resilience or benefit-finding. We also do not have data on SES status, although we believe patients' educational background is a reliable proxy for this component of SES. In addition, the measures used in the study, the Distress Thermometer and HADS, have not been specifically validated in a sample of AfricanAmerican with prostate cancer, as they are used here, and the study was conducted from a site with a low concentration of African-American men, who may therefore be exceptional and not representative of men from community health centers with a different demographic. Future research is needed to clarify the type and extent of distress in AfricanAmerican men with prostate cancer; what factors are moderators of this distress; and if AfricanAmerican are in fact more resilient when confronted with prostate cancer, why this is so.

In light of the nuances of African-American culture discussed above, it is possible that AfricanAmericans have a qualitatively distinct perspective of the cancer experience that fosters different coping mechanisms than those of other groups. Future studies including measures of benefit-finding as well as qualitative questions about the cancer experience would be one way to begin learning about these mechanisms.

\section{Acknowledgements}

This research was supported by the T.J. Martell Foundation.

\section{References}

1. ACS. 2008.

2. Zigmond AS, Snaith RP. The hospital anxiety and depression scale. Acta Psychiatr Scand 1983;67(6):361-370.

3. Roth AJ, Kornblith AB, Batel-Copel L et al. Rapid screening for psychologic distress in men with prostate carcinoma: a pilot study. Cancer 1998;82(10):1904-1908.

4. Derogatis LLR, Melisaratos NN. The brief symptom inventory: an introductory report. Psychol med 1983; 13(3):595.

5. Zabora J, BrintzenhofeSzoc K, Curbow B, Hooker C, Piantadosi S. The prevalence of psychological distress by cancer site. Psycho-Oncology 2001;10(1):19-28.

6. Farber JM. Psychosocial distress in oncology outpatients. J Psychosoc Oncol 1984;2(3-4):109.

7. McDaniel JS, Musselman DL, Porter MR, Reed DA, Nemeroff CB. Depression in patients with cancer. Diagnosis, biology, and treatment. Arch Gen Psychiatry 1995;52(2):89-99.

8. Sellick SM, Crooks DL. Depression and cancer: an appraisal of the literature for prevalence, detection, and practice guideline development for psychological interventions. Psycho-Oncology 1999;8(4):315-333.

9. Stefanek ME, Derogatis LP, Shaw A. Psychological distress among oncology outpatients. Prevalence and severity as measured with the Brief Symptom Inventory. Psychosomatics 1987;28(10):530-532, 537-539.

10. Roth AP, Passik S. Anxiety in men with prostate cancer may interfere with effective management of the disease. Prim Care Cancer 1996;16:30.

11. ACS. 2006.

12. Anai S, Pendleton J, Wludyka P et al. Prostate cancer screening and detection in inner-city and underserved men. J Natl Med Assoc 2006;98(4):515-519.

13. Wingo PPA, Ries LLA, Parker SSL, Heath CCW. Long-term cancer patient survival in the United States. Cancer Epidemiol Biomarkers Prev 1998;7(4):271.

14. Smith GE, DeHaven MJ, Grundig JP, Wilson GR. African-American males and prostate cancer: assessing knowledge levels in the community. $J$ Natl Med Assoc 1997;89(6):387-391.

15. Ford ME, Vernon SW, Havstad SL, Thomas SA, Davis SD. Factors influencing behavioral intention regarding prostate cancer screening among older African-American men. $J$ Natl Med Assoc 2006;98(4): 505-514.

16. Forrester-Anderson IT. Prostate cancer screening perceptions, knowledge and behaviors among African American men: focus group findings. $J$ Health Care Poor Underserved 2005;16(4 Suppl A):22-30.

17. Tannor BB, Ross L. Physician-patient discussions about prostate-specific antigen test use among AfricanAmerican men. J Natl Med Assoc 2006;98(4):532-538.

18. Woods VD, Montgomery SB, Belliard JC, RamirezJohnson J, Wilson CM. Culture, black men, and 
prostate cancer: what is reality? Cancer Control 2004;11(6):388-396.

19. Demark-Wahnefried WW, Strigo TT, Catoe KK et al. Knowledge, beliefs, and prior screening behavior among blacks and whites reporting for prostate cancer screening. Urology 1995;46(3):346.

20. Parker PA, Cohen L, Bhadkamkar VA et al. Demographic and past screening behaviors of men attending a free community screening program for prostate cancer. Health Promot Pract 2006;7(2):213-220.

21. Abrams H, Doogan R, Lampert Hill H, Rosenbaum C. The emotional impact of cancer. In Osteen B, Cady B, Rosenthal P (eds.). Cancer Manual, American Cancer Society, Boston, MA, 1990.

22. Rosenfeld B, Roth AJ, Gandhi S, Penson D. Differences in health-related quality of life of prostate cancer patients based on stage of cancer. Psycho-Oncology 2004;13(11):800-807.

23. Lubeck DDP, Kim HH, Grossfeld GG et al. Health related quality of life differences between black and white men with prostate cancer: data from the cancer of the prostate strategic urologic research endeavor. J Urol 2001;166(6):2281

24. Jenkins RR, Schover LRLR, Fouladi RTRT et al. Sexuality and health-related quality of life after prostate cancer in African-American and white men treated for localized disease. J Sex Marital Ther 2004;30(2):79.

25. Jacobsen PB. Screening for psychological distress in cancer patients: challenges and opportunities. $J$ Clin Oncol 2007;25(29):4526-4527.

26. Esper P, Mo F, Chodak G et al. Measuring quality of life in men with prostate cancer using the functional assessment of cancer therapy-prostate instrument. Urology 1997;50(6):920-928.

27. Christenfeld NJ, Sloan RP, Carrol D, Greenland S. Risk factors, confounding, and the illusion of statistical control. Psychosom Med 2004;66(6):868-875.

28. Sachs-Ericsson N, Plant EA, Blazer DG. Racial differences in the frequency of depressive symptoms among community dwelling elders: the role of socioeconomic factors. Aging Ment Health 2005;9(3):201-209.

29. Zigmond AS, Snaith RP. The hospital anxiety and depression scale. Acta Psychiatr Scand 1983;67(6): 361-370.

30. Cella DDF, Tulsky DDS, Gray GG et al. The functional assessment of cancer therapy scale: development and validation of the general measure. $J$ Clin Oncol 1993;11(3):570.

31. Esper PP, Mo FF, Chodak GG et al. Measuring quality of life in men with prostate cancer using the functional assessment of cancer therapy-prostate instrument. Urology 1997;50(6):920.

32. Jacobsen PB, Donovan KA, Trask PC et al. Screening for psychologic distress in ambulatory cancer patients. Cancer 2005;103(7):1494-1502.

33. Tuinman MA, Gazendam-Donofrio SM, HoekstraWeebers JE. Screening and referral for psychosocial distress in oncologic practice: use of the distress thermometer. Cancer 2008;113(4):870-878.

34. Spinhoven P, Ormel J, Sloekers PP et al. A validation study of the Hospital Anxiety and Depression Scale (HADS) in different groups of Dutch subjects. Psychol Med 1997;27(2):363-370.

35. Savard J, Laberge B, Gauthier JG, Ivers H, Bergeron MG. Evaluating anxiety and depression in HIV-infected patients. J Pers Assess 1998;71(3):349-367.

36. Singer S, Kuhnt S, Gotze $\mathrm{H}$ et al. Hospital anxiety and depression scale cutoff scores for cancer patients in acute care. Br J Cancer 2009;100(6):908-912.
37. Castelli L, Binaschi L, Caldera P, Torta R. Depression in lung cancer patients: is the HADS an effective screening tool? Support Care Cancer 2009.

38. Bjelland I, Dahl AA, Haug TT, Neckelmann D. The validity of the Hospital Anxiety and Depression Scale. An updated literature review. $J$ Psychosom Res 2002;52(2):69-77.

39. Walker J, Postma K, McHugh GS et al. Performance of the Hospital Anxiety and Depression Scale as a screening tool for major depressive disorder in cancer patients. J Psychosom Res 2007;63(1):83-91.

40. Baker F. Diagnosing depression in African Americans. Community Ment Health J 2001;37(1):31-38.

41. Kessler R. Depression screening. $J$ Fam Pract 2003;52(6):466; author reply 467.

42. Magai C, Kerns M, Consedine NS, Fyffe D. Depression in older ethnic groups: a test of the generality of the social precursors model. Res Aging 2003;25(2):144-171.

43. Gray-Little B, Hafdahl AR. Factors influencing racial comparisons of self-esteem: a quantitative review. Psychol Bull 2000;126(1):26-54.

44. Twenge JM, Crocker J. Race and self-esteem: metaanalyses comparing whites, blacks, Hispanics, Asians, and American Indians and comment on Gray-Little and Hafdahl (2000). Psychol Bull 2002;128(3):371-408; discussion 409-420.

45. Connell CM, Gibson GD. Racial, ethnic, and cultural differences in dementia caregiving: review and analysis. Gerontologist 1997;37(3):355-364.

46. Hughes SL, Giobbie-Hurder A, Weaver FM, Kubal JD, Henderson W. Relationship between caregiver burden and health-related quality of life. Gerontologist 1999; 39(5):534-545

47. Plant EA, Sachs-Ericsson N. Racial and ethnic differences in depression: the roles of social support and meeting basic needs. J Consult Clin Psychol 2004;72(1): $41-52$.

48. Kleinman A, Good B (eds). Culture and Depression, University of California Press: Berkeley, CA, 1985.

49. Consedine NS, Magai C, Cohen CI, Gillespie M. Ethnic variation in the impact of negative affect and emotion inhibition on the health of older adults. $J$ Gerontol B Psychol Sci Soc Sci 2002;57(5):396-408.

50. Consedine NS, Magai C, Horton D. Ethnic variation in the impact of emotion and emotion regulation on health: a replication and extension. J Gerontol B Psychol Sci Soc Sci 2005;60(4):165-173.

51. Gallo LC, Matthews KA. Do negative emotions mediate the association between socioeconomic status and health? Ann N Y Acad Sci 1999;896:226-245.

52. Steward RJ, Jo HI, Murray D et al. Psychological adjustment and coping styles of urban African American high school students. J Multicultural Couns Dev 1998;26(2):70-82.

53. Thorson JA, Powell FC, Sarmany-Schuller I, Hampes WP. Psychological health and sense of humor. J Clin Psychol 1997;53(6):605-619.

54. Cummings SM, Neff JA, Husaini BA. Functional impairment as a predictor of depressive symptomatology: the role of race, religiosity, and social support. Health Soc Work 2003;28(1):23-32.

55. True G, Phipps EJ, Braitman LE et al. Treatment preferences and advance care planning at end of life: the role of ethnicity and spiritual coping in cancer patients. Ann Behav Med 2005;30(2):174-179.

56. Musick MA, Koenig HG, Hays JC, Cohen HJ. Religious activity and depression among communitydwelling elderly persons with cancer: the moderating effect of race. J Gerontol B Psychol Sci Soc Sci 1998;53(4):S218-S227. 
57. Utsey S, Giesbrecht N, Hook J, Stanard P. Cultural, sociofamilial and psychological resources that inhibit psychological distress in African Americans exposed to stressful life events and race related stress. J Couns Psychol 2008;55(1):49-62.

58. Becker G, Newsom E. Resilience in the face of serious illness among chronically ill African Americans in later life. J Gerontol B Psychol Sci Soc Sci 2005;60(4): S214-S223.
59. Zuvekas SH, Fleishman JA. Self-rated mental health and $\mathrm{racial} / \mathrm{ethnic}$ disparities in mental health service use. Med Care 2008;46(9):915-923.

60. Anglin DM, Alberti PM, Link BG, Phelan JC. Racial differences in beliefs about the effectiveness and necessity of mental health treatment. Am J Community Psychol 2008;42(1-2):17-24.

61. Cruz M, Pincus HA, Harman JS, Reynolds CF 3rd, Post EP. Barriers to care-seeking for depressed African Americans. Int J Psychiatry Med 2008;38(1):71-80. 
Copyright of Psycho-Oncology is the property of John Wiley \& Sons, Inc. and its content may not be copied or emailed to multiple sites or posted to a listserv without the copyright holder's express written permission.

However, users may print, download, or email articles for individual use. 